\title{
Visual monitoring of the melting front propagation in a paraffin- based PCM
}

\author{
Pavel Charvát, Josef Štětina, Tomáš Mauder ${ }^{*}$ and Lubomír Klimeš \\ Faculty of Mechanical Engineering, Brno University of Technology, 61669 Brno, Czech Republic
}

\begin{abstract}
Experiments were carried out in an environmental chamber with the aim to monitor the melting front propagation in a rectangular cavity filled with a paraffin-based Phase Change Material (PCM). The PCM was contained in transparent containers with the heat flux introduced by means of an electric heating element. The stabilized power source was used to maintain the constant heat output of the heating elements. The experiments were performed for the heat flux introduced at the side wall of the container and at the upper surface of the PCM. The paraffin-based PCM RT28HC with the phase change temperature of $28{ }^{\circ} \mathrm{C}$ was used in the experiments. The temperature in the environmental chamber was maintained at the melting temperature of the PCM. The propagation of the melting front was monitored with a digital camera and temperatures at several locations were monitored with RTDs and thermocouples. Significant natural convection was observed for the heat flux introduced at the side wall of the container. As a result the melting front propagated much faster at the top of the container than at its bottom. The heat flux introduced at the upper-surface of the PCM resulted in almost one-dimensional propagation of the melting front. The acquired data are to be used for validation of an in-house developed numerical model based on the fronttracking method.
\end{abstract}

\section{Introduction}

Thermal energy storage makes it possible to balance supply and demand of thermal energy over certain periods of time. Thermal energy storage (TES) is used in heating and cooling applications in order to increase the overall efficiency of these systems. At present, most thermal energy storage systems use sensible heat storage materials, such as water, to store thermal energy. Latent heat thermal energy storage (LHTES), which employs phase change of matter in order to store thermal energy, is much less commonly used. The phase change from solid to liquid state and vice versa is most commonly used in LHTES. The materials for LHTES employing solid-liquid phase change are called Phase Change Materials.

The advantages of LHTES in comparison to sensible heat storage are the reason for a widespread research in this area [1]. One of the advantages of LHTES over sensible heat storage is the high energy storage density in narrow temperature intervals. However, most PCMs have relatively low thermal conductivity and thus large heat exchange areas are needed to achieve high heat transfer rates between the heat transfer fluid and the PCM. Full scale LHTES units can contain large volumes of phase change materials. The melting and solidification of a PCM does not take place in the entire volume at the same time but rather the melting or solidification front starts from the heat exchange surface and then propagates from the surface into the volume of the PCM.
Melting of PCMs in the enclosures or containers of various shapes has been studied by many researchers in the last decade. There are two cases of melting in the enclosures - unconstrained melting and constrained melting. Unconstrained melting means that the solid PCM is not held in place and it can freely move within the enclosure or container due to gravity force or other forces. Since the PCMs have higher density in the solid state than in the liquid state the unconstrained solid PCM tends to sink to the bottom of the enclosure. In case of the constrained melting some measures are taken to prevent solid PCM from moving freely in the enclosure or container.

Kamkari et al. [2] reported experimental investigation of the effect of inclination angle on convection-driven melting of phase change material in a rectangular enclosure. Lauric acid was used as a PCM in the experiments. One of the walls of the enclosure was heated (at constant temperature) while other walls were thermally insulated. Three wall temperatures $\left(55^{\circ} \mathrm{C}\right.$, $60^{\circ} \mathrm{C}$ and $\left.70{ }^{\circ} \mathrm{C}\right)$ and three inclination angles $\left(0^{\circ}, 45^{\circ}\right.$, and $90^{\circ}$ ) were studied. At $0^{\circ}$ inclination the heated wall was horizontal (at the bottom of the cavity) and at $90^{\circ}$ inclination the heated wall was vertical. The shortest melting time was achieved for $0^{\circ}$ inclination for all three wall temperatures.

Shmueli et al. [3] carried out numerical investigation of melting in a vertical cylindrical tube. Previously obtained experimental results were used for verification of the simulation results. A commercially available PCM

Corresponding author: mauder@fme.vutbr.cz 
RT27 (Rubitherm $\mathrm{GmbH}$ ), with the melting interval from $26{ }^{\circ} \mathrm{C}$ to $28{ }^{\circ} \mathrm{C}$ was used in the study. The authors report good quantitative agreement between the simulation and the experimental results in terms of the melt fraction as a function of time.

Fan et al. [4] reported experimental and numerical investigation of constrained melting heat transfer of a PCM in a circumferentially finned spherical capsule. The authors used a Plexiglas capsule with the inner diameter of $100 \mathrm{~mm}$ submerged in the Plexiglas water tank to allow for the visual observation. A transparent tube connected to the top of the sphere was used for indirect measurement of melt fraction based on the volumetric expansion of the PCM during melting. The PCM used in the study was n-octadecane $\left(\mathrm{C}_{18} \mathrm{H}_{38}\right)$, with the nominal melting range from $28{ }^{\circ} \mathrm{C}$ to $30{ }^{\circ} \mathrm{C}$. A thin straight circumferential fin was introduced along the equator of the capsule for augmentation of heat transfer.

The present paper deals with the visual monitoring of melting front propagation in paraffin-based PCM. The goal of the experiments was to obtain experimental data for validation of numerical models.

\section{Experimental set-up}

The goal of the experiments was to obtain experimental data for validation of in-house numerical models that are developed for simulation of melting front propagation in PCMs and other materials. Melting of a PCM in the enclosed cavity represents one of the simplest cases of melting front propagation. The real-life LHTES units often employ PCM-fluid heat exchangers with rather complex heat exchange surfaces where many melting fronts can propagate simultaneously in the volume of the LHTES unit.

\subsection{Phase Change Material}

Alkanes (paraffins) represent relatively non-expensive PCMs with the melting range suitable for thermal energy storage in building thermal systems. The alkanes are a group of hydrocarbons with the chemical formula $\mathrm{C}_{\mathrm{n}} \mathrm{H}_{\mathrm{n}+2}$. The melting temperature of an alkane depends on the number of carbon atoms. The melting temperature of alkanes generally increases with the increasing number of carbon atoms. Many producers of phase change materials use paraffin as the basic component in the so-called paraffin-based PCMs. Other components are usually added in order to modify the properties of the PCM.

The experiments presented in this paper were conducted in an environmental chamber. The environmental chamber allowed for the control of the ambient temperature, which was an important parameter in the experiments. The environmental chamber was a large one with the lengths of $8.85 \mathrm{~m}$, width of $5 \mathrm{~m}$ and height of $3.8 \mathrm{~m}$. The dimensions of the chamber allowed for the installation of all experimental equipment inside of the chamber. It was also possible for the investigators to be present in the chamber during the experiments or to periodically check on the progress of the experiments.
For that reason several PCMs with the melting temperature between $15{ }^{\circ} \mathrm{C}$ and $35{ }^{\circ} \mathrm{C}$ were selected as suitable for the experiments. The properties of the paraffin-based PCM RT28HC that was used for the experiments reported in this paper are shown in Table 1.

Table 1. Properties of RT28HC

\begin{tabular}{|c|c|}
\hline Melting area & $\begin{array}{c}27-29^{\circ} \mathrm{C} \\
\text { main peak } 28^{\circ} \mathrm{C}\end{array}$ \\
\hline Congealing area & $\begin{array}{c}29-27^{\circ} \mathrm{C} \\
\text { main peak } 27^{\circ} \mathrm{C}\end{array}$ \\
\hline $\begin{array}{c}\text { Heat storage capacity } \pm 7.5 \% \\
\text { (Combination of latent and sensible heat } \\
\left.\text { in a temperature range of } 21^{\circ} \mathrm{C} \text { to } 36^{\circ} \mathrm{C}\right)\end{array}$ & $245 \mathrm{~kJ}$ \\
\hline Specific heat capacity & $2 \mathrm{~kJ} \cdot \mathrm{kg}^{-1} \mathrm{~K}^{-1}$ \\
\hline Density solid $\left(15^{\circ} \mathrm{C}\right)$ & $880 \mathrm{~kg}^{-3}$ \\
\hline Density liquid $\left(40^{\circ} \mathrm{C}\right)$ & $770 \mathrm{kgm}^{-3}$ \\
\hline Heat conductivity & $0.2 \mathrm{~W} \cdot \mathrm{m}^{-1} \mathrm{~K}^{-1}$ \\
\hline Volume expansion & $12.5 \%$ \\
\hline Maximum operation temperature & $50{ }^{\circ} \mathrm{C}$ \\
\hline
\end{tabular}

The data sheets of the PCMs mostly contain just the basic thermophysical properties such as the density in the solid and liquid state, thermal conductivity, specific heat, etc. Some characteristics, such as viscosity of a PCM in the liquid state as a function of temperature, which is an important input for CFD simulations, are very often difficult to obtain.

The phase change of most PCMs is not isothermal but it takes place in a certain temperature interval, as can be seen in Table 1. Latent heat, by definition, is the heat absorbed or released by matter at constant temperature (usually associated with the change of state or phase). To avoid confusion, some producers of PCMs provide heat storage capacity in a certain temperature interval around the phase change temperature instead of the latent heat.

The temperature-enthalpy curve is another way of providing information about the thermal capacity of a PCM in relation to temperature. The temperatureenthalpy curves are usually obtained by means of Differential Scanning Calorimetry where a small sample of PCM (usually a few milligrams) is heated up and cooled down at the given rate (e.g. $1 \mathrm{~K}$ per minute).

As the DSC uses very small samples of the PCM the method is suitable for microencapsulated PCMs were capsules hold very small amounts of PCM [5]. The microencapsulated phase change materials are often used in building applications as they can be mixed with construction materials such as concrete or plaster [6]. Other methods, such as T-history [7], were proposed for larger samples of PCMs.

\subsection{Containers for the PCMs}

In order to monitor the propagation of the melting front the PCM was contained in transparent containers during the experiments. The containers were made of $25 \mathrm{~mm}$ thick PMMA. An aluminium plate with the attached 
electric heating elements was used to introduce the heat flux to the block of PCM. The first set of experiments was performed with the heat flux introduced on the side wall of the container. The shape and dimensions of the container for these experiments can be seen in Fig. 1.

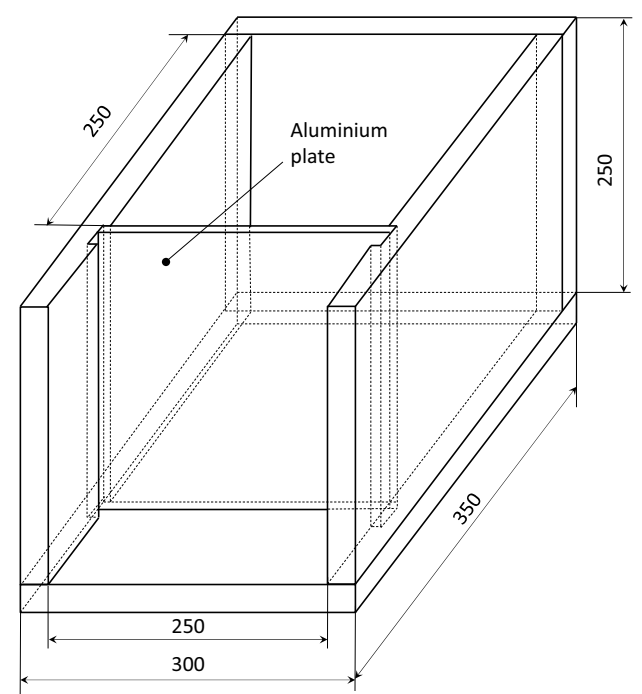

Fig. 1. Container with a heated side wall.

As the heat flux introduced on the side wall of the container led to a significant natural convection in the melted PCM, a container for the heating from the uppersurface was prepared. The aluminium plate in this case was $10 \mathrm{~mm}$ thick and it had the dimensions to fit into the container. It was important to make sure that the heating plate was in good contact with the PCM during the experiment.

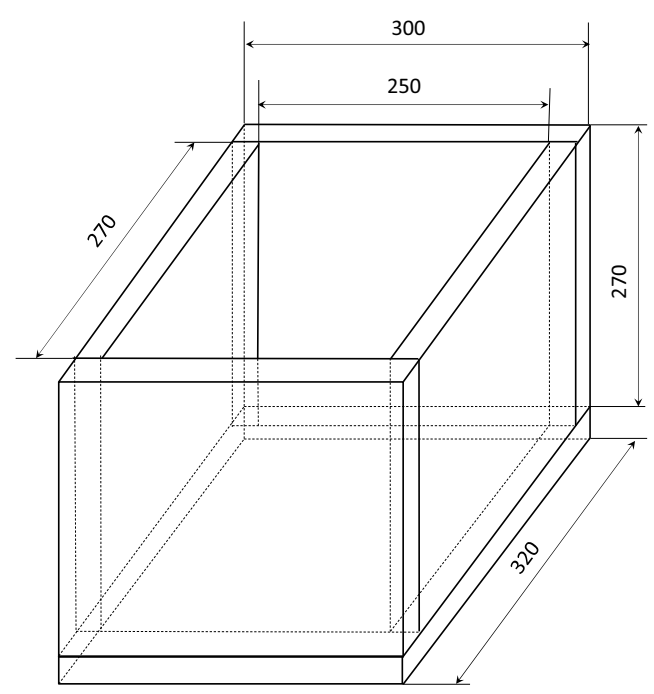

Fig. 2. Container for heating at the upper-surface.

\subsection{Monitoring technique}

The monitoring of the melting front propagation was done with the use of a digital camera. The progress of the melting front was relatively slow and it was sufficient to take pictures in 1 minute intervals in case of the side-wall heating and 5 minute intervals in case of the upper-surface heating. The pictures were taken with the Nikon D90 camera fitted with the Sigma macro lens The Aputure Pro Coworker II AP-WTR3N trigger was used to synchronize photo shooting with data acquisition. As the experiments took place in the environmental chamber an LED flash was used for the illumination of the containers.

The environmental chamber had stainless steel walls that created disturbing reflections of the photo flash. For that reason the container was placed in a photo shooting tent with the opaque black background (Fig. 3).

The data acquisition system for temperature measurements was based on the CompactDAQ Chassis connected to a laptop computer via an USB port. The NI 9219 module with four analogue channels was used for connecting the RTD (Pt100) temperature sensors. The Pt100 temperature sensors were used for the monitoring of the aluminium plate temperature. Thermocouples were used for monitoring of the PCM temperature at various positions. The positions of thermocouples differed in the side-wall and upper-surface heating case.

\section{Results}

An in-house image processing code was used for conversion of the photos into black and white ( 2 bit) images to make the propagation of the melting front better visible and also to assess the melt fraction.

It was assumed that the heat flux introduced on one side of the PCM block would lead to the quasi-2D propagation of the melting front. This assumption was confirmed by the experiments. However, it was important that the entire block of the PCM would have approximately the uniform temperature at the beginning of the experiment. The liquid PCM was poured into the container and then the container was placed in the environmental chamber where the ambient temperature was kept below the melting temperature. It could take two or more days for the PCM to fully solidify and to reach uniform temperature in the entire volume. The ambient temperature in the environmental chamber was set to $27^{\circ} \mathrm{C}$ during the experiments.

\subsection{Side-wall heating}

The experiment with the heat flux introduced at the side-wall of the container can be seen in Fig. 3. As could be seen in Fig. 1 one wall of the container was made of an aluminium plate. The electric heating foil and thermal insulation were placed on the external side of the aluminium plate. The temperature of the PCM was measured with the Teflon coated T-type thermocouples at 5 locations at the midplane of the PCM block. Brass tubes were used to hold the thermocouples at the desired positions. The junctions of the thermocouples protruded a few millimetres from the tubes in order to be in the direct contact with the PCM. The thermocouples (sensing junctions) were positioned at the middle of the height of the container. The first thermocouple was 24 
$\mathrm{mm}$ from the heated wall and the other four were $39 \mathrm{~mm}$, $54 \mathrm{~mm}, 69 \mathrm{~mm}$ and $84 \mathrm{~mm}$ from the heated wall (the aluminium plate with the electric heating foil). Fig. 3 shows a situation where 4 thermocouples are in the melted PCM while the fifth thermocouple is still in the solid PCM.

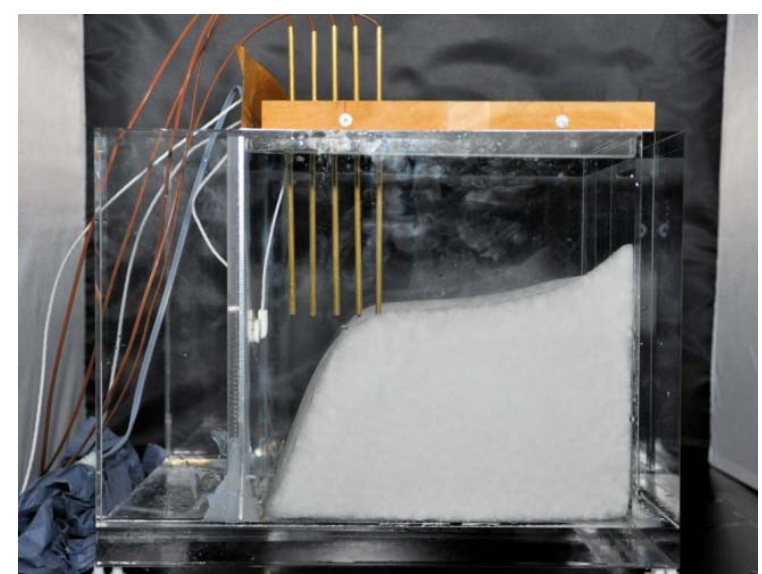

Fig. 3. Experiment with side-wall heating

The temperatures recorded in one of the experiments can be seen in Fig. 4. Though the power supplied to the heater was constant $(30 \mathrm{~W})$ the surface temperature of the heater $T_{\text {surface }}$ changed throughout the experiment. The temperature of the melted PCM increased during the experiment but was almost the same (thermocouples $\mathrm{T}_{1}$ to $\mathrm{T}_{4}$ ) due to natural convection. The temperature measured by the thermocouple $T_{5}$ does not change very much as the thermocouple was buried in the solid PCM. As can be seen in Fig. 4 the initial temperature of the PCM block was not uniform (temperatures at time $0 \mathrm{~s}$ ).

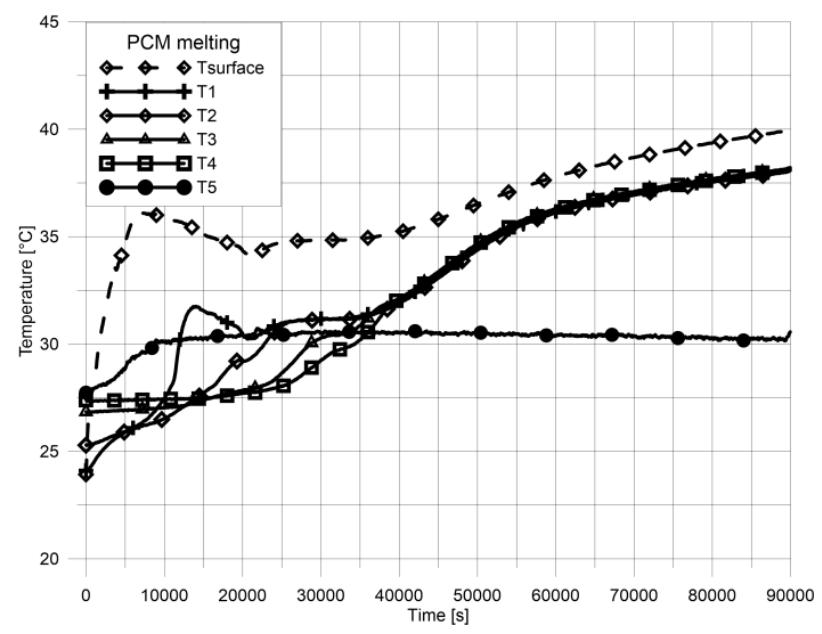

Fig. 4. Temperatures for side-wall heating

Propagation of the melting front can be seen in Fig. 5. Black regions represent the $\mathrm{PCM}$ in the liquid state and the white regions are the PCM in the solid state. The natural convection in the liquid state significantly contributed to the melting process. As could be seen in Fig. 5 the heat flux introduced at the side wall of the container induced significant natural convection in the liquid PCM and as a result the melting front propagated much faster at the top of the container than at its bottom.

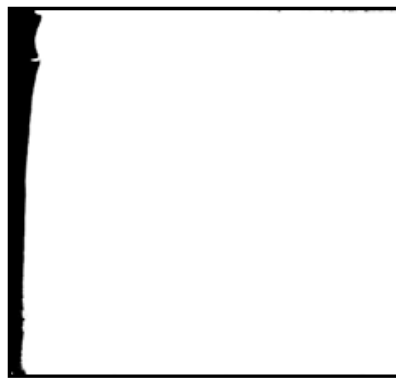

time $=6000 \mathrm{~s}$

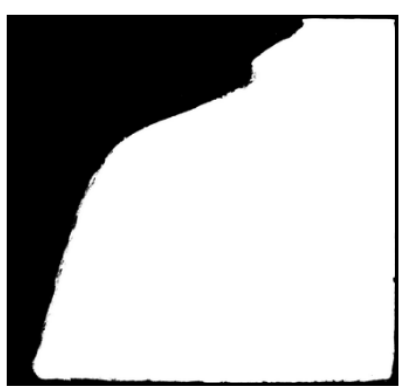

time $=35000 \mathrm{~s}$

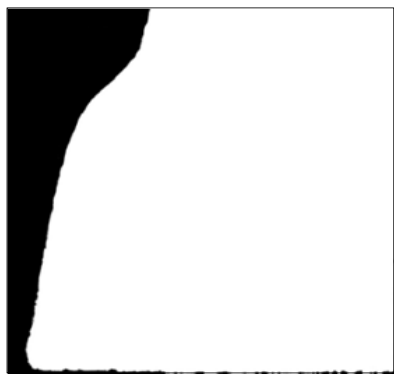

time $=20000 \mathrm{~s}$

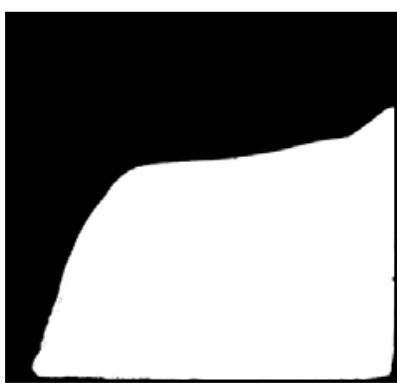

time $=50000 \mathrm{~s}$
Fig. 5. Melting front propagation (side-wall heating)

\subsection{Upper-surface heating}

When the heater is positioned at the top of the container natural convection is significantly reduced and as a result the melting front propagates much slower than in case of the side-wall heating. The photo of the experiment with the heating plate at the upper surface of the PCM is shown in Fig. 6. The melting process in this case is almost one-dimensional and the melting front is a horizontal plane.

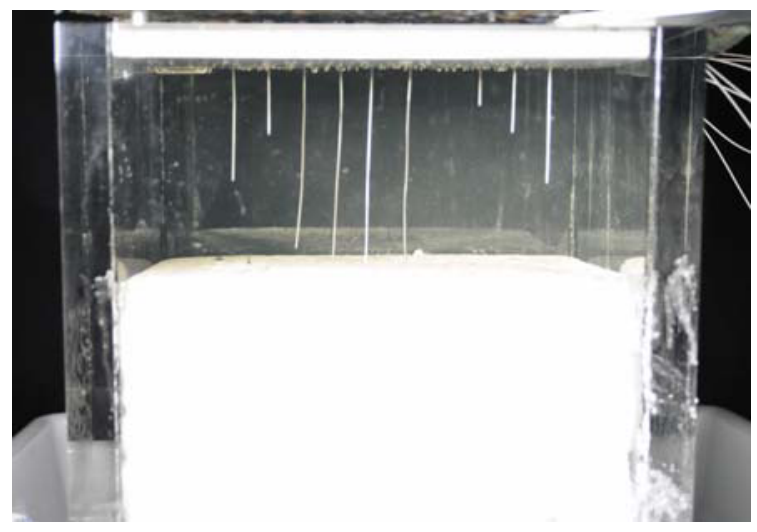

Fig. 6. Experiment with upper-surface heating

As in case of the side-wall heating the PCM temperature at various positions (distances from the aluminium plate) was monitored with thermocouples. The thermocouples were located in the vertical midplane of the container. The plot of the temperatures can be 
seen in Fig. 7. The surface temperature of the aluminium plate was higher than in case of the side-wall heating. Different heating elements were used in this case and the heating power was $60 \mathrm{~W}$. In spite of that the melting front propagated much slower in case of the uppersurface heating as natural convection in melted PCM was significantly suppressed.

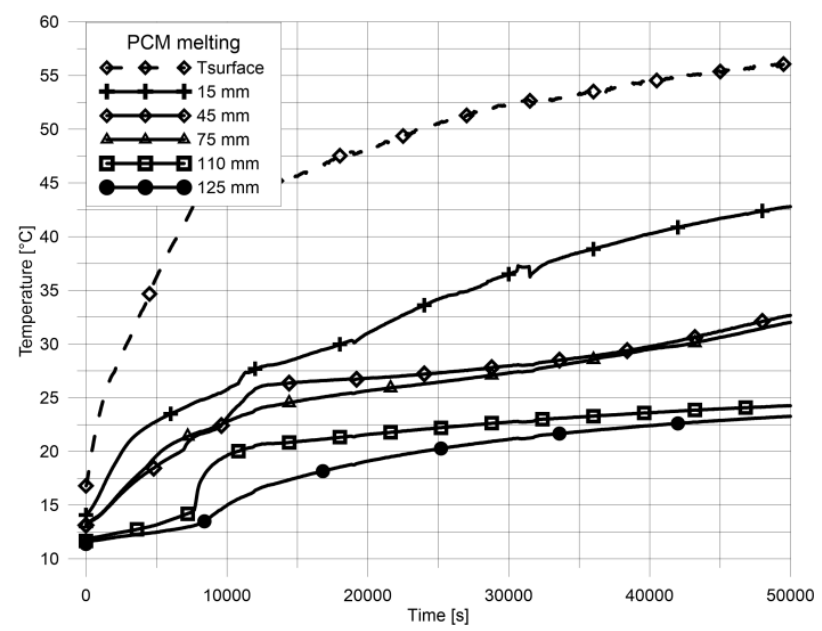

Fig. 7. Temperatures for upper-surface heating

The melting front propagation is shown in Fig. 8. The melt fraction at particular time intervals is much smaller than in case of the side-wall heating.
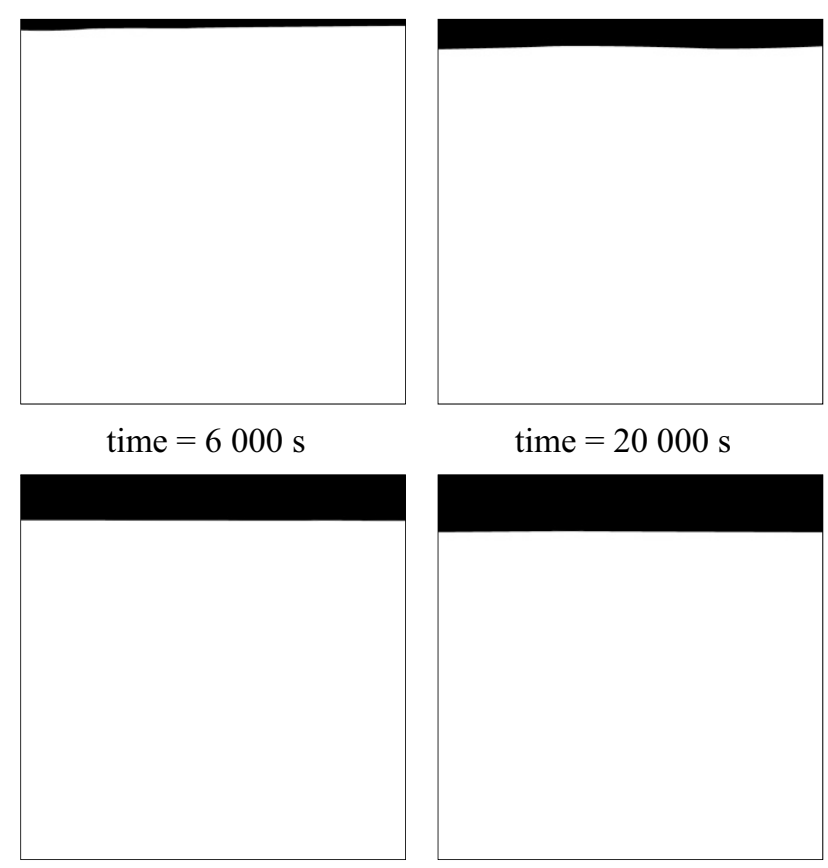

time $=35000 \mathrm{~s}$

time $=50000 \mathrm{~s}$

Fig. 8. Melting front propagation (upper-surface heating)

\subsection{Melt fractions}

The melt fractions for the two experiments are shown in Table 2. The melt fraction is the ratio of the PCM in the liquid state to the total volume of PCM. As can be seen in Table 2 the melt fraction at each time interval is much higher in case of the side-wall heating than in case of the upper-surface heating. Such result is not surprising as the heat flux direction from the top down is the worst case scenario. The experiment with the heat flux introduced at the bottom of the container is under preparation. As reported by Kamkari et al. [2] the heat flux introduced at the bottom of the enclosure should result in the largest melt fractions at the given time intervals.

Table 2. Melt fractions

\begin{tabular}{|c|c|c|}
\hline \multirow{2}{*}{ Time [s] } & \multicolumn{2}{|c|}{ Melt fraction [-] } \\
\cline { 2 - 3 } & side-wall heating & upper-surface heating \\
\hline 6000 & 0.0435 & 0.0228 \\
\hline 20000 & 0.1636 & 0.0740 \\
\hline 35000 & 0.2949 & 0.1181 \\
\hline 50000 & 0.4763 & 0.1474 \\
\hline
\end{tabular}

\section{Conclusions}

Monitoring of the melting front in the paraffin-based PCM was done with the use of a digital camera and image processing code. Two cases were studied: heat flux introduced at one side of the PCM block and the heat flux introduced at the upper surface of PCM block. Significant natural convection was observed in case of the heat flux introduced at the side wall of the container. As a result the melting front propagated much faster at the top of the container than at its bottom. The heat flux introduced at the upper-surface of the PCM resulted in almost one-dimensional propagation of the melting front. The acquired experimental data will be used in the development of in-house codes for simulation of melting front propagation.

This work was supported by the Czech Science Foundation under contract GA15-11977S.

\section{References}

1. F. Agyenim, N. Hewitt, P. Eames, M. Smyth, Renew. and Sustain. Energy Reviews 14 (2010)

2. B. Kamkari, H. Shokouhmand, F. Bruno, Int. J. of Heat and Mass Transfer 72 pp. 186-200 (2014)

3. H. Shmueli, G. Ziskind, R. Letan, International Journal of Heat and Mass Transfer 53 pp. 40824091 (2010)

4. L-W. Fan, Z-Q. Zhu, S-L. Xiao, M-J. Liu, H. Lu, Y. Zeng, Z-T. Yu, K-F. Cen, Applied Thermal Engineering 100 pp. 1063-1075 (2016)

5. M. Ostry, R. Prikryl, P. Charvat, T. Mlcoch, B. Bakajova, Materiali in Tehnologije, 46, pp. 813-816 (2012)

6. M. Ostry, P. Charvat, Procedia Engineering, 57, 2013, pp. 837-843 (2013)

7. H. Hong, S. K. Kim, Y-S. Kim, Int. J. of Refrigeration 27 pp. 360-366 (2004) 